\title{
REPLANTEAMIENTO DE UN TEMA CONTROVERTIDO. GÉNESIS Y RETENCIÓN DEL DOBLE POSESIVO EN EL ESPAÑOL ANDINO
}

\author{
GERMÁN DE GRANDA \\ Universidad de Valladolid
}

Muy recientemente Beatriz Garza Cuarón exponía, en un acertado e inteligente trabajo, algunas de las peculiaridades originales y valiosas de la lingüística iberoamericana desde su comienzo, en el siglo pasado, hasta la actualidad ${ }^{1}$.

Es obligado reconocer, sin embargo, que, junto con las notas positivas y enriquecedoras que la mencionada especialista tan justamente recoge y glosa, también se dan, en este campo de estudio, otras características metodológicas que son, contrariamente (y por desgracia), negativas e indeseables y ello no sólo por su erróneo o parcializado planteamiento teórico sino, y aún más, por los devastadores efectos que la aplicación de las mismas al estudio de problemas concretos puede determinar.

Entre estas pautas de índole claramente rechazable se debe incluir, a mi parecer en una posición muy destacada, la sistemática contraposición, intensamente ideologizada en muchas ocasiones, de dos posturas apriorísticas (las que podríamos denominar respectivamente «indigenista» e «hispanista») en el examen de cualquier problema lingüístico del español americano que, por indefinición (mayor o menor e incluso, a veces, inexistente) de alguno de sus elementos caracterizadores específicos, pueda dar oportunidad para ello.

Esta lamentable actitud respecto a la determinación de la matriz genética de rasgos del español americano que deberían ser analizados, en su totalidad, de modo desapasionado y carente de apriorismos subjetivos ha sido mencionada en los últimos tiempos, con evidente preocupación dadas sus

\footnotetext{
${ }^{1}$ B. Garza Cuarón, «Originalidades de la lingüística en Iberoamérica», en Scripta Philo-
} logica In Honorem Juan M. Lope Blanch, II, México, 1992, págs. 439-451. 
posibles implicaciones perturbadoras de todo tipo, por varios estudiosos de nuestra área de trabajo como, entre otros, Klaus Zimmermann ${ }^{2}$ y Luis Fernando Lara quien, aunque refiriéndose en concreto a la fonética del español de América Central y México, alude sin duda a un estado de cosas mucho más general cuando dedica al tema las siguientes (muy justas) consideraciones: «La influencia de las lenguas amerindias sobre la fonética del español ha sido largamente debatida. En muchos trabajos los resultados de la investigación de esa influencia están determinados por una selección ideológica previa que divide a los investigadores en dos bandos: el de los que tratan de demostrar que lo que ha dado sus características principales al español de esta región es la influencia de las lenguas amerindias de sustrato - en especial el nahuatl- y el de los que se proponen demostrar que tal influencia ha sido mínima»» ${ }^{3}$.

Deseo dedicar estas páginas al examen, realizado (al menos es mi propósito) con la máxima objetividad, de un rasgo lingüístico que ha sido hasta ahora, como otros muchos del español americano, analizado frecuentemente a partir de condicionamientos metodológicos parcializados (en el sentido mencionado en los párrafos anteriores) $\mathrm{y}$, por ello, deformadores (en una dirección teórica o en la opuesta) de su auténtica realidad tal como ella es rastreable a través de datos difícilmente refutables por su consistencia empírica.

Me refiero, en concreto, al denominado doble posesivo o posesivo redundante existente en el área lingüística andina y caracterizable por la doble marcación en superficie de la relación de posesión que, en español general, es expresada por una sola marca morfosintáctica.

La modalidad más extendida de esta estructura gramatical es, sin duda, la referida a construcciones del tipo de su amiga de Juan, su casa de mi papá que se encuentran ampliamente difundidas en el español de Perú ${ }^{4}$, de Bolivia ${ }^{5} \mathrm{e}$, incluso, del noroeste argentino ${ }^{6}$.

\footnotetext{
${ }^{2} \mathrm{~K}$. Zimmermann, «Aspectos teóricos y metodológicos de la investigación sobre el contacto de lenguas en Hispanoamérica», en K. Zimmermann (ed.), Lenguas en contacto en Hispanoamérica, Frankfurt am Main, 1995, págs. 9-34. Véase también R. Maldonado Soto, «Entre indigenistas, hispanistas y sustratos», en Nueva Antropología, 6, 1983, págs. 119-132.

${ }^{3}$ L. F. Lara, "Áreas lingüísticas. VII: México y América Central», en G. Holtus, M. Metzeltin y Ch. Schmidt (eds.), Lexikon der Romanistischen Linguistik, VI, 1, Tübingen, 1992, págs. 559-567 (pág. 563).

${ }^{4}$ Cfr. R. Cerrón-Palomino, «Enseñanza del castellano: deslindes y perspectivas», en A. Escobar (ed.), El reto del multilingüismo en el Perú, Lima, 1972, págs. 143-166 y "Calcos sintácticos en el castellano andino», en San Marcos, 14, 1976, págs. 93-101.

${ }^{5}$ Cfr. J. G. Mendoza, «Aspectos del castellano hablado en Bolivia», en Historia y presente del español de América, Valladolid, 1992, págs. 437-499 (pág. 485).

6 Véase especialmente M. Fleming de Cornejo, Relatos folklóricos salteños, Salta, 1988, transcripción de textos grabados en trabajo de campo (especialmente págs. $45,75,84$, etc.).
} 
Respecto a este tipo particular de doble posesivo (que, como veremos, no es sino una clase especial de un fenómeno global mucho más complejo) se han mantenido, reproduciendo así en relación con este rasgo concreto del español andino la sistemática contraposición de enfoques «indigenistas» e «hispanistas», dos tesis explicativas genéticas polarmente enfrentadas: la del mantenimiento de un arcaísmo castellano, expresada por Inés Pozzi-Escot ${ }^{7}$ $\mathrm{y}$, más matizadamente, por J. A. Rodríguez Garrido ${ }^{8}$ y la de transferencia, por contacto con el español local, de una estructura morfosintáctica quechua, postulada por Anthony Lozano ${ }^{9}$ quien afirma, tajantemente, que tal construcción «... can be traced directly to Quechua interference» ${ }^{10}$. Ambas tomas de posición pueden, sin duda, alegar argumentos a su favor.

Como apoyo a la hipótesis de la retención, en la zona andina, de un rasgo sintáctico del español medieval y clásico deben ser tomados en cuenta tanto los ejemplos medievales (sos fijas del Campeador en el Poema del Cid) como, sobre todo, los que facilita Keniston ${ }^{11}$ con referencia al siglo XVI (¿tan sabia era su madre del señor? en Lope de Rueda, queremos sigir sus consejos de Cristo en Santa Teresa, sus padres della en Ginés Pérez de Hita, que es la obra propia suya de Cristo en Fray Luis de León, ¿sabes si es hija suya de aquel Pascual Crespo? en Lope de Rueda, etc.) ${ }^{12}$ que, en conjunto, demuestran la vigencia en castellano, hasta el Siglo de Oro, de una doble marcación de la posesión si bien limitada, exclusivamente, a construcciones de tercera persona posesora ${ }^{13}$. Que, por otra parte, esta estructura sintáctica llegó a América en el habla de sus conquistadores y primeros pobladores es, desde luego, indudable. Véanse, para comprobarlo, los pasajes en que la usan, por ejemplo, Diego de Ordaz (Alonsico, su hijo de Herrera, os encomiendo mucho) ${ }^{14}$, Inés García de Santiago, moradora de la localidad peruana de Cañete, en 1594 ([F. de Soto] la hiço donaçion de diez iseis mill pesos corrientes iensu testamento de Soto diçen dejo mandado sele cumpliesen ipaguen...) ${ }^{15}$ o, por transmisión directa del

\footnotetext{
${ }^{7}$ I. Pozzi-Escot, Apuntes sobre el castellano de Ayacucho, Lima, 1973 (pág. 3).

8 J. A. Rodríguez Garrido, «Sobre el uso del posesivo redundante en el español del Perú», en Lexis, 6, 1982, págs. 117-123.

9 A. Lozano, "Syntactic borrowing in Spanish from Quechua. The noun phrase», en Lingüistica e indigenismo moderno en América, Lima, 1975, págs. 297-305.

${ }^{10}$ Artículo citado en la nota anterior, pág. 298.

11 H. Keniston, The Syntax of Castilian Prose. The Sixteenth Century, Chicago, 1937.

12 Obra citada en la nota anterior, pág. 244.

13 Ibidem. Véase ahora, sobre este tema, C. Company, «Semántica y sintaxis de los posesivos duplicados en el español de los siglos Xv y XVI», en Romance Philology, 48, 1994, págs. 111-135.

14 J. M. Lope Blanch, El habla de Diego de Ordaz, México, 1985 (pág. 186).

${ }^{15}$ Cfr. M. B. Fontanella de Weinberg (ed.), Documentos para la historia lingüistica de Hispanoamérica. Siglos XVI a XVIII, Madrid, 1993 (pág. 156).
} 
habla de los españoles y criollos del área, el mismo Guamán Poma (... y dejó al sol en su testamento por heredero de todos sus bienes que había) ${ }^{16}$.

Enfrentadamente, la hipótesis de la transferencia desde el quechua al español del área del rasgo que aquí nos ocupa puede basarse en la estrecha similitud que el mismo posee respecto a las estructuras morfosintácticas de posesión existentes en aquel código de comunicación indoamericano.

En efecto, las reglas de formación de la frase genitiva en quechua (y, también, en aimara) ${ }^{17}$ son básicamente las siguientes: anteposición obligatoria del elemento posesor al poseído, marcación obligatoria de aquél por un elemento sufijado, invariable, indicador de relación posesiva y, finalmente, marcación del segundo constituyente (poseído) por elementos sufijados, variables, indicadores de la persona del posesor (y, mayoritariamente, coincidentes con los morfemas verbales actanciales correspondientes) ${ }^{18}$. Téngase debidamente en cuenta, sin embargo, que las realizaciones de superficie derivadas de la aplicación de las dos últimas reglas mencionadas presentan una apreciable variación diatópica la cual, por otra parte, no altera (salvo en un solo caso que posteriormente mencionaremos) su homogeneidad tipológica de base.

Así, el elemento invariable postpuesto al lexema indicador del posesor es $-p a$ en quechua ayacuchano y santiagueño, $-q$ (realizado como [x])/$q p a$ en cuzqueño, -pak/-bak (genitivo-benefactivo) en quechua ecuatoriano y $-p /-p a$ en casi todo el resto de las modalidades dialectales de dicha lengua ( $-p$ tras vocal, $-p a$ tras consonante) ${ }^{19}$. Del mismo modo, el sufijo, indicador de la persona del poseedor, que se postpone al segundo constituyente (poseído) de la construcción genitiva quechua coincide, en algunos casos, diatópicamente en la totalidad de las áreas dialectales (-yki para segunda persona, - $n$ para tercera) pero no así en otros (alargamiento de vocal para primera persona en Quechua I frente a $-y$ en Quechua II, $-k u n a$ en Quechua I frente a $-y k u$ en Quechua II; para la cuarta persona exclusiva, etc.) ${ }^{20}$.

Ahora bien, el fenómeno del doble posesivo en la zona andina no se reduce a la estructura, de tercera persona poseedora, su casa de mi papá o su amigo de Juan, única de que aquí nos hemos ocupado hasta ahora. Abarca también otras modalidades que han sido, respectivamente, puestas de relie-

\footnotetext{
${ }^{16}$ F. Guamán Poma de Ayala, Nueva crónica y buen gobierno, Madrid, 1987 (pág. 133).

${ }^{17}$ Sobre esta última lengua véase E. H. Martín, «Lenguas en contacto. Acerca del uso de los posesivos en el español andino», en Actas del Segundo Congreso Nacional de Lingüistica, II, San Juan (Argentina), 1986, págs. 289-292.

${ }^{18}$ Cfr. R. Cerrón-Palomino, Quechumara, La Paz, 1994, págs. 151-152.

19 Véase especialmente R. Cerrón-Palomino, Lingüistica quechua, Cuzco, 1987, págs. 134-135.

${ }^{20}$ Obra citada en la nota anterior, págs. 137-145 y 303-308.
} 
ve por investigadores con Clodoaldo Soto ${ }^{21}$, Liliana Minaya ${ }^{22}$, Luis Miran$\mathrm{da}^{23}$, Rodolfo Cerrón-Palomino ${ }^{24} \mathrm{y}$, en trabajos de síntesis recientes, consideradas globalmente por Anna María Escobar ${ }^{25}$.

Las más relevantes de entre ellas son las referidas a estructuras posesivas (siempre con marcación doble de esta relación) caracterizadas por la imposición del orden poseedor-poseído (de mi padrino su carro, de mi mi papá es carnicero), coincidente con el que se da obligatoriamente en quechua y las que, con poseedor de primera o segunda persona (no de tercera), expresan doblemente la relación de posesión, bien por medio de la reiteración del adjetivo posesivo (antepuesto y postpuesto al constituyente poseído) como en esta es tu hoja tuya, bien por el empleo de adjetivo posesivo y construcción de + poseedor (de mí mi papá es carnicero, mi santo de mi lo han celebrado).

La consideración conjunta, desde un punto de vista genético, de la totalidad de las estructuras de doble marcación de la relación posesiva existente en el español andino requiere, en primer lugar, una cuidadosa tarea de diferenciación de las mismas en cuanto a su origen causal.

En primer lugar, la inexistencia en la lengua histórica española de estructuras posesivas del tipo de las caracterizadas por el orden sintáctico posesor-poseido (de mi papá su casa, de Pedro su mujer) o por la reiteración de marcas de posesión cuando el posesor es de primera o segunda persona (esta es tu hoja tuya, mi santo de mi lo han celebrado) las cuales, por otra parte, coinciden plenamente con los resultados de la aplicación de las reglas de formación de sintagmas posesivos existentes en la lengua quechua (y en la aimara) no deja otra posibilidad de explicación causal de las mismas que considerarlas, de modo exclusivo, como transferencias al español andino de rasgos gramaticales propios de aquellas lenguas amerindias.

Muy otra es, desde luego, la situación que comporta la doble marcación de la relación posesiva en los sintagmas del español andino en que el po-

\footnotetext{
${ }^{21}$ C. Soto, «La interferencia quechua-español. Una doble perspectiva», en Lingüistica y educación. Actas del IV Congreso de ALFAL, Lima, 1978, págs. 619-626.

${ }^{22}$ L. Minaya, «Descripción de estructuras sintácticas del habla del niño ayacuchano», en el volumen mencionado en la nota anterior, págs. 464-477.

${ }^{23}$ L. Miranda, «Peculiaridades sintácticas en el español de los niños de zonas de influencia quechua» en el volumen citado en la nota 21 , págs. 478-483.

${ }^{24} \mathrm{R}$. Cerrón-Palomino, «Aspectos sociolingüísticos y pedagógicos de la motosidad en el Perú», en R. Cerrón-Palomino y G. Solís Fonseca (eds.), Temas de lingüistica amerindia, Lima, 1990, págs. 153-180.

${ }_{25}$ A. M. Escobar, «El español andino y el español bilingüe. Semejanzas y diferencias en el uso del posesivo», en Lexis, 16, 1992, págs. 189-222 y «Andean Spanish and bilingual Spanish: Linguistic characteristics», en P. Cole, G. Hermon y M. D. Martín (eds.), Language in the Andes, Newark, 1994, págs. 51-73.
} 
sesor es determinable como de tercera persona (su libro de él, sus problemas de San Juan).

La existencia, en este caso, de estructuras homólogas tanto en el español del siglo XVI como en quechua (y aimara) requiere, a mi parecer, como primer paso para una consideración adecuada del tema la aplicación al mismo de la tesis explicativa de la causación múltiple (hispano-quechua/aimara) enfocándose, así, el rasgo en cuestión del español andino como producto, conjunto, tanto de la retención de modalidades sintácticas del español clásico como de transferencia al castellano local de condicionamientos gramaticales (coincidentes con aquéllas) presentes en las lenguas amerindias de contacto de la zona andina ${ }^{26 .}$

Ésta ha sido, en efecto, la postura teórica adoptada al respecto, con todo acierto, por José Luis Rivarola ${ }^{27} \mathrm{y}$, al sumarnos aquí a ella, debemos valorar debidamente el hecho de que la misma integra armoniosamente, despojándolas de su exclusivismo parcializado, las tesis explicativas (que antes hemos caracterizado respectivamente como «indigenista» e «hispanista») mantenidas en relación con este problema por estudiosos como Anthony Lozano, Inés Pozzi-Escot, J. A. Rodríguez Garrido, etc.

No es, sin embargo, suficiente (al menos en mi opinión) el haber identificado la causación múltiple - en este caso doble - del origen del rasgo lingüístico andino que en estas páginas consideramos. Es preciso plantearse, inmediatamente, otro interrogante, referido ahora no a la génesis causal de dicho fenómeno gramatical sino a su mantenimiento hasta la actualidad: ¿cuál ha sido el factor determinador de, o, en todo caso, preponderante en la retención del doble posesivo en el español del área andina?

Es esta cuestión, aparentemente, de difícil respuesta pero que creo puede recibir adecuada aclaración considerando las implicaciones metodológicas que, al respecto, son deducibles del examen de dos hechos (de fisonomía no sólo diferente sino contrapuesta pero de sentido análogo) que se dan en los dos territorios extremos, meridional y septentrional, del área lingüística andina y que, hasta ahora, no han sido correctamente relacionados con el problema que nos ocupa.

\footnotetext{
${ }^{26}$ Sobre el concepto teórico de causación múltiple véanse Y. Malkiel, «Multiple versus simple causation in linguistic change», en To Honor Roman Jakobson, II, La Haya, 1967, págs. 1228-1246 y «On hierarchyzing the components of multiple causation», en Studies in Language, 1, 1977, págs. 81-108.

Un ejemplo de aplicación de este principio metodológico al análisis de fenómenos lingüísticos del español americano puede verse en G. de Granda «De nuevo sobre la causación múltiple en el español de América (a propósito de dos rasgos morfosintácticos del español paraguayo)», en Scripta Philologica In Honorem Juan M. Lope Blanch, II, México, 1992, págs. 491-506.

27 J. L. Rivarola, La formación lingüistica de Hispanoamérica, Lima, 1990 (pág. 180).
} 
Se trata, en concreto, de la inexistencia de la doble marca de posesivo en el español ecuatoriano, dato mencionado recientemente por P. Muysken ${ }^{28}$ y mucho antes por $\mathrm{H}$. Toscano ${ }^{29}$, y, enfrentadamente, de la presencia, hasta hoy ${ }^{30}$, de tal rasgo en el noroeste de la Argentina, lo que contrasta vivamente con su total desuso en el resto del territorio de dicha República ${ }^{31}$.

La primera circunstancia mencionada debe ser conectada causalmente, sin lugar a dudas, con una notable peculiaridad de la modalidad diatópica de quechua utilizado en el Ecuador (y en el ingano de Colombia y el napeño del Perú) respecto a la expresión sintáctica de la posesión.

Me refiero, obviamente, a que en estos dialectos quechuas (y sólo én ellos) la posesión se expresa con una única marca (la invariable postpuesta al posesor) que es el morfema genitivo-benefactivo -pak/-bak exceptuándose, además, incluso de esta regla reductora la construcción con primera persona posesora, caso en el que ni el constituyente posesor ni el constituyente poseído llevan marca alguna ${ }^{32}$.

Mientras que la segunda, es decir la referida al uso de doble posesivo en el español argentino exclusivamente en su área noroeste, sólo es explicable, desde el punto de vista genético, si se valora debidamente la total coincidencia geográfica entre dicha zona y los territorios que, hasta principios del siglo $\mathrm{XIX}^{33}$, manejaron variantes de quechua que, aunque al parecer ligeramente diferentes entre sí ${ }^{34}$, poseían todas ellas (en cuanto al punto que aquí nos interesa) doble marcación de la posesión nominal como, aún hoy, ocurre en su última modalidad subsistente, el quechua de Santiago del Estero ${ }^{35}$.

Con base en los dos hechos considerados no parece que pueda ser apreciada como controvertible la deducción de que, puesto que el doble posesivo del español andino se da exclusivamente cuando (y sólo cuando) el castellano local está (o estuvo, como en el noroeste argentino) en contacto con

\footnotetext{
${ }^{28} \mathrm{P}$. Muysken, «The Spanish that quechua speakers learn: L2 learning as norm-governed behavior», en R. Andersen (ed.), Second Languages. A Crosslinguistic Perspective, Rowley, 1984, págs. 101-124.

${ }^{29} \mathrm{H}$. Toscano, El español en el Ecuador, Madrid, 1953.

${ }^{30}$ Fundamento esta afirmación en mi conocimiento directo del habla local. Pero véase también la obra mencionada en la nota 6 .

${ }^{31}$ Cfr. B. E. Vidal de Battini, El español de la Argentina, Buenos Aires, 1964.

${ }^{32}$ R. Cerrón-Palomino, obra citada en la nota 19, pág. 198.

33 Véase, sobre este tema, G. de Granda, "Quechua y español en el noroeste argentino. Una precisión y dos interrogantes», en Lexis, 17, 1993, págs. 259-274.

${ }^{34}$ Cfr. R. L. J. Nardi, «El quichua de Catamarca y La Rioja», en Cuadernos del Instituto Nacional de Investigaciones Folklóricas (Buenos Aires), 3, 1962, págs. 189-285 y W. F. Adelaar, «Raíces lingüísticas del quechua de Santiago del Estero», en Actas de las Segundas Jornadas de Lingüistica Aborigen, Buenos Aires, 1995, págs. 25-50.

${ }^{35}$ Cfr. Domingo A. Bravo, Estado actual del Quichua santiagueño, Tucumán, 1965.
} 
variedades diatópicas del quechua $-\mathrm{y}$ del aimara - poseedoras de similar marcación doble de la relación en cuestión y, por el contrario, no es empleado en áreas donde no se manejó históricamente la lengua quechua (Argentina, excepto su área noroccidental) o donde la misma (Ecuador, Sur de Colombia, etc.) no poseía en su estructura gramatical la expresión doblemente marcada de la posesión, debe ser apreciado, sin duda, como factor determinador ( $\mathrm{o}$, al menos, preponderante) de la retención, hasta hoy, del doble posesivo en el español andino no la preservación de un arcaísmo castellano, sino, más bien, el contacto (histórico o actual) con las lenguas quechua y aimara y el consiguiente reforzamiento, por ello, de aquellas estructuras castellanas que, como la que aquí estudiamos, existen homólogamente en estas últimas.

Considero que con los materiales aportados en estas páginas queda, al menos en propósito, cumplido el objetivo que nos habíamos propuesto al proyectarlas, es decir, el replanteamiento desde un enfoque explicativo objetivo, de índole genética, de la presencia de construcciones gramaticales caracterizadas por una doble marcación sintáctica de la relación de posesión en el español del área lingüística andina. Pero, antes de dar por finalizada nuestra presente tarea, debo expresar dos precisiones finales, de carácter sociolingüístico la primera, de perfil diatópico la segunda.

Consiste aquélla en manifestar mi total disconformidad con los datos presentados por A. M. Escobar como resultado de su intento de atribuir, basándose en un reducidísimo corpus de encuestas, determinados tipos de construcciones con doble posesivo (concretamente las estructuradas por medio de adj. pos. $+\mathrm{N}+$ de + pron. de $1 .^{a}$ y $2 .^{a}$ personas, como en mi santo de mí, o a través de adj. pos. + $\mathrm{N}+$ adj. pos., como en esta es tu hoja tuya) sólo al español bilingüe (es decir, al manejado por hablantes maternos de quechua y sólo consecutivamente de español) y no al español andino (utilizado por hablantes maternos de castellano) ${ }^{36}$.

Como indica, muy justamente, al respecto Rocío Caravedo ${ }^{37}$, desde un enfoque sociolingüístico globalizador «... no se justifica la separación entre el español de bilingües y el de monolingües...» porque «muchos fenómenos [del español bilingüe]... se expanden en la interacción comunicativa de contacto entre diversos grupos y empiezan a usarse incluso entre los hablantes con dominio amplio de español» ${ }^{38}$.

Este último punto de vista, opuesto al mantenido por A. M. Escobar, no

${ }^{36}$ Cfr. el trabajo mencionado en primer lugar en la nota 25 (pág. 211).

${ }^{37}$ R. Caravedo, «Espacio geográfico y modalidades lingüísticas en el español de Perú», en Historia y presente del español de América, Valladolid, 1992, págs. 719-741.

38 Trabajo mencionado en la nota anterior, págs. 729-730. 
sólo es apoyado por datos aportados, al respecto, en varios trabajos por R. Cerrón-Palomino ${ }^{39}$ referidos al español peruano sino que recibe una sorprendente confirmación en el hecho, comprobado por mí mismo en trabajo de campo, de que en el área de Salta (noroeste argentino), en que la población de las zonas centrales de la misma es, con toda probabilidad ${ }^{40}$, monolingüe en español desde hace casi dos siglos, se emplean aún, con cierto vigor y en zonas próximas incluso a la ciudad capital, construcciones como ahora vamo a comé'l bollo de mi ávio mio ${ }^{41}$, atribuidas por A. M. Escobar, como hemos visto, con exclusividad al español bilingüe de la zona andina.

La precisión (final) de índole diatópica consiste en resaltar una vez más (después de haberlo hecho ya repetidas veces tanto en el texto de este trabajo como en su mismo título) que las conclusiones aquí postuladas respecto a la génesis y el mantenimiento hasta la actualidad de estructuras sintácticas españolas caracterizadas por el uso de marca posesiva doble o reiterada son aplicables solamente a la situación vigente en el área lingüística andina $\mathrm{y}$ de ningún modo a otras zonas dialectales del español americano.

Deseo, con ello, diferenciar claramente el fenómeno gramatical de la doble marcación de la relación de posesión tal como se presenta en la zona de Los Andes, desde el sur de Colombia hasta el noroeste argentino, del (solamente hasta cierto punto homólogo al anterior) que parece darse en el español mexicano ${ }^{42}$, respecto al cual (y en particular a su extensión creciente) se han postulado recientemente factores determinadores de índole pragmática ${ }^{43}$ que no parecen guardar relación alguna de semejanza con los que han actuado (y actúan aún) en el área andina como determinadores del fenómeno específico aquí considerado.

\footnotetext{
${ }^{39}$ Véase, por ejemplo, el mencionado en la nota 24 (págs. 165-168).

${ }^{40}$ Cfr. mi trabajo citado en la nota 33 (pág. 263).

${ }^{41}$ Obra citada en la nota 6 (pág. 84). La informante a quien pertenece la frase citada en el texto fue Dorotea Tolaba de Borja, de 76 años, quien vivía en 1976-1983 en Potrero de Uriburu, Departamento de la Capital (Salta).

${ }^{42}$ Cfr. Juan M. Lope Blanch, «La influencia del sustrato en la gramática del español mexicano», en el volumen del autor Estudios sobre el español de México, México, 1972, págs. 157-164 (págs. 160-161) y José G. Moreno de Alba, Minucias del lenguaje, México, 1987 (págs. 64-65).

${ }^{43}$ Véanse Erica C. García, «Frecuencia relativa de uso como síntoma de estrategias etnopragmáticas», en K. Zimmermann (ed.), Lenguas en contacto en Hispanoamérica, Frankfurt am Main, 1995, págs. 51-71 (pág. 57) y, de Concepción Company Company, «Su casa de Juan: estructura y evolución de la duplicación posesiva en español», en Actas del I Congreso Internacional Anglo-Hispano, Madrid, 1994, págs. 73-88 y "Cantidad vs. cualidad en el contacto de lenguas. Una incursión metodológica en los posesivos 'redundantes' del español americano», en Nueva Revista de Filología Hispánica, 43, 1995, págs. 315-339.
} 